\title{
Towards the development of improved tests for negative symptoms of schizophrenia in a validated animal model
}

DOI:

10.1016/j.bbr.2016.06.021

\section{Document Version}

Accepted author manuscript

Link to publication record in Manchester Research Explorer

\section{Citation for published version (APA):}

Sahin, C., Doostdar, N., \& Neill, J. (2016). Towards the development of improved tests for negative symptoms of schizophrenia in a validated animal model. Behavioural brain research, 312, 93-101.

https://doi.org/10.1016/j.bbr.2016.06.021

\section{Published in:}

Behavioural brain research

\section{Citing this paper}

Please note that where the full-text provided on Manchester Research Explorer is the Author Accepted Manuscript or Proof version this may differ from the final Published version. If citing, it is advised that you check and use the publisher's definitive version.

\section{General rights}

Copyright and moral rights for the publications made accessible in the Research Explorer are retained by the authors and/or other copyright owners and it is a condition of accessing publications that users recognise and abide by the legal requirements associated with these rights.

\section{Takedown policy}

If you believe that this document breaches copyright please refer to the University of Manchester's Takedown Procedures [http://man.ac.uk/04Y6Bo] or contact uml.scholarlycommunications@manchester.ac.uk providing relevant details, so we can investigate your claim.

\section{OPEN ACCESS}




\section{Accepted Manuscript}

Title: Towards the development of improved tests for negative symptoms of schizophrenia in a validated animal model

Author: Ceren Sahin Nazanin Doostdar Joanna C. Neill

PII: $\quad$ S0166-4328(16)30375-8

DOI: $\quad$ http://dx.doi.org/doi:10.1016/j.bbr.2016.06.021

Reference: $\quad$ BBR 10264

To appear in: $\quad$ Behavioural Brain Research

Received date: $\quad 11-5-2016$

Revised date: $\quad$ 9-6-2016

Accepted date: $\quad 11-6-2016$

Please cite this article as: Sahin Ceren, Doostdar Nazanin, Neill Joanna C.Towards the development of improved tests for negative symptoms of schizophrenia in a validated animal model.Behavioural Brain Research http://dx.doi.org/10.1016/j.bbr.2016.06.021

This is a PDF file of an unedited manuscript that has been accepted for publication. As a service to our customers we are providing this early version of the manuscript. The manuscript will undergo copyediting, typesetting, and review of the resulting proof before it is published in its final form. Please note that during the production process errors may be discovered which could affect the content, and all legal disclaimers that apply to the journal pertain. 


\section{Towards the development of improved tests for negative symptoms of schizophrenia in a validated animal model}

Ceren Sahin ${ }^{1}$, Nazanin Doostdar ${ }^{2}$, Joanna C. Neill ${ }^{2 *}$.

${ }^{1}$ Marmara University, Faculty of Pharmacy, Department of Pharmacology and Psychopharmacology Research Unit, Istanbul, 34668, Turkey

${ }^{2}$ Manchester Pharmacy School, University of Manchester, Oxford Rd, M13 9PT, UK

*Corresponding author: Prof Jo Neill (joanna.neill@manchester.ac.uk)

Manchester Pharmacy School, University of Manchester, Oxford Road, Manchester, M13 9PT, UK. Tel.: 01612754538

E-mail: joanna.neill@manchester.ac.uk 


\title{
Highlights
}

- Sub-chronic PCP treated rats show reduced optimism in response to ambiguous environmental situations in an optimistic bias paradigm.

- Sub-chronic PCP treated rats show indifference towards a high-value reward in comparison to a low-value reward in an affective bias paradigm.

- These behavioural tests in this animal model for schizophrenia could be useful in evaluating the efficacy of novel compounds targeting negative symptoms of schizophrenia.

\begin{abstract}
Negative symptoms in schizophrenia remain an unmet clinical need. There is no licensed treatment specifically for this debilitating aspect of the disorder and effect sizes of new therapies are too small to make an impact on quality of life and function. Negative symptoms are multifactorial but often considered in terms of two domains, expressive deficit incorporating blunted affect and poverty of speech and avolition incorporating asociality and lack of drive. There is a clear need for improved understanding of the neurobiology of negative symptoms which can be enabled through the use of carefully validated animal models. While there are several tests for assessing sociability in animals, tests for blunted affect in schizophrenia are currently lacking. Two paradigms have recently been developed for assessing negative affect of relevance to depression in rats. Here we assess their utility for studying negative symptoms in schizophrenia using our well validated model for schizophrenia of sub-chronic (sc) treatment with Phencyclidine (PCP) in adult female rats. Results demonstrate that sc PCP treatment produces a significant negative affect bias in response to a high value reward in the optimistic and affective bias tests. Our results are not easily explained by the known cognitive deficits induced by sc PCP and support the hypothesis of a negative affective bias in this model. We suggest that further refinement of these two tests will provide a means to investigate the neurobiological basis of negative affect in schizophrenia, thus supporting the assessment of efficacy of new targets for this currently untreated symptom domain.
\end{abstract}


Keywords: Schizophrenia, negative symptoms, animal model, NMDA receptor, PCP, affective and cognitive bias

\section{INTRODUCTION}

Despite the wide range of antipsychotic medication available for the treatment of schizophrenia, negative symptoms remain an unmet clinical need (Tsapakis et al., 2015). A recent large meta-analysis of 168 studies demonstrated small effect sizes of current therapeutic strategies, ranging from atypical antipsychotics and antidepressants to psychological interventions, that failed to reach the threshold for clinically meaningful improvement (Fusar-Poli et al., 2015). Persistent negative symptoms have a substantial impact on patients' functional outcome, leading to disability and reduced quality of life (Foussias et al., 2015). Davis and colleagues provide a recent update on new pharmacological treatments for negative symptoms including examining important methodological consideration for future clinical trials (Davis et al., 2014). Recent on-going clinical trials are reviewed in Arango et al., (2013). However in spite of recent advances in this area, effective therapies are still lacking. Carefully validated animal models combined with appropriate tests for negative symptoms in animals form a critical part of the development of new therapeutic strategies for negative symptoms (Barnes et al., 2014a; Neill et al., 2014). However, many aspects of negative symptom domains have been poorly investigated in animal studies and when they have, the most successful work has been conducted on the social withdrawal domain in rats (Gururajan et al., 2010; Moser, 2014; Neill et al., 2010, 2014; Wilson and Koenig, 2014). These studies are certainly valuable and of ethological relevance in such a gregarious species. However such studies lack analysis of emotional regulation or affective state, processes essential for effective human social interaction and communication. There have been some attempts to investigate anticipatory motivation in animals, but the relevance of these tests for schizophrenia symptomatology is debatable (Barnes et al., 2014a). Perhaps surprisingly, the other debilitating aspects of negative symptoms have almost never been investigated in a well-validated animal model for schizophrenia. That is of course partly due to the uniquely human characteristics of certain domains such as poverty of speech and blunted affect. However, there is still a major gap in the animal studies addressing other aspects of negative symptoms and with carefully developed tests even these aspects may be 
studied. In order to improve our understanding of the neurobiological mechanisms underlying negative symptom domains and therefore develop improved treatment strategies, it is essential to assess these using carefully constructed test procedures in well validated animal models for the disorder.

Although negative symptoms are defined by five consensus-based domains, two have been especially considered as determinant factors in terms of best describing this facet of the illness. The concept is called the 'two factor model'. One of the factors in this model reflects 'diminished motivation and pleasure' and the other is related to 'diminished verbal/nonverbal expression or communicative output' (Kring and Barch, 2014) describing avolition and expressive deficit domains. As we suggested in our 2014 review, there is a clear argument for separate tests for each specific domain of negative symptoms (Neill et al., 2014) as MATRICS have recommended for cognition (Young et al., 2009). Sub-chronic (sc) treatment with phencyclidine (PCP) and other N-methyl-D-aspartate receptor (NMDAR) antagonists have been widely used in an attempt to mimic certain behavioural and neuropathological deficits observed in schizophrenia (Meltzer, 2013; Morris et al., 2005; Neill et al., 2010, 2014) with animals tested at least 7 days following the PCP treatment regimen. We have demonstrated that these deficits are reversed by several novel targets and low doses of atypical antipsychotics (Grayson et al. 2015, Leger et al. 2015, Neill et al. 2010, 2014). When it comes to investigating negative symptoms, we and others have shown that sc PCP induces social withdrawal but not anhedonia in rodents (Lydall et al., 2010; Neill et al., 2014) with the growing consensus that anhedonia per se. is not a feature of negative symptoms (Barnes et al., 2014a; Foussias et al., 2014) lending further support for the validity of this model. However, there have been no studies to date demonstrating the effect of sc treatment with PCP or other NMDAR antagonists, probably the best validated animal model for schizophrenia, albeit still requiring further validation, on anticipatory pleasure/motivation or on affective state. Therefore, we aimed to investigate the effect of our sc PCP treatment regime ( $2 \mathrm{mg} / \mathrm{kg}$ i.p. twice daily for 7 days followed by 7 days washout) on anticipatory motivation and affective state in female rats. To address this issue, we employed two different paradigms: affective and cognitive bias tests. To our knowledge, this is the first study attempting to model anticipatory reward and affect in an animal model for schizophrenia. 
The task of optimistic cognitive bias used in our study was developed by Brydges et al. (2011) based on earlier studies with starlings (Brilot et al., 2009, 2010; Matheson et al., 2008). The rationale for the task developed by Brydges et al. (2011) and variations used by other laboratories, was based on Harding's (2004) original study. In this study, the concept of cognitive/judgement bias, defined as the propensity of a subject to show behaviour indicating anticipation of either relatively positive or negative outcomes in response to ambiguous stimuli (Mendl et al., 2009) was first introduced in animals (Harding et al., 2004). In their work, Brydges et al. (2011) devised an ethologically relevant methodology for investigating the effect of environmental enrichment on optimistic cognitive bias in rats. They showed that rats transferred from un-enriched to enriched conditions gave more optimistic responses to an ambiguous stimulus than control rats maintained in un-enriched conditions. They argued that testing cognitive bias in this way could provide information on an animal's emotional state and that this method could be used to develop novel therapeutic strategies for mood disorders. We have adapted this test to assess optimistic cognitive bias in our animal model for schizophrenia, sc PCP treatment.

Recently Emma Robinson's laboratory (2013) developed a novel behavioral test to measure changes in the affective state of rodents. This test, known as the Affective Bias Test (ABT) is another ethologically relevant test in which animals form an association between an odour and food reward. In this study, strength and valence (positive or negative) of this relationship is altered using pharmacological and environmental manipulations. Replicating findings in healthy human volunteers, this study showed that acute treatment with typical (ie fluoxetine, citalopram) and atypical antidepressants (ie agomelatine, mirtazapine) induces a significant positive affective bias in healthy rats. Furthermore, certain drugs associated with inducing negative affective state in humans (eg the anxiogenic agent, FG7142) also induced a negative affective bias in rats as indicated by significantly fewer choices for the odour paired with these agents. This paradigm was also found to be sensitive to manipulation of the absolute reward value. These findings support the translational and predictive validity of the ABT (Stuart et al., 2013).

Our overall aim in this study is to investigate the potential of these two tests to assess affect and anticipatory reward deficits of relevance to schizophrenia. In order to achieve this aim, we adapted Brydges's paradigm to investigate the effect of our sc PCP treatment regimen on anticipatory behaviour of rats in a task of optimistic cognitive bias. Secondly, we investigated 
effects of sc PCP treatment on affective bias using the ABT. Due to the success of our initial study, we believe we have identified two paradigms that could be used with some further adaptations to enhance understanding of these two distinct aspects of negative symptom domains and assist in the development of new therapies. These tests could also be used to assess affective bias and anticipatory motivation in various animal models for other CNS disorders (Knuesel et al., 2014). However these paradigms have certain limitations that should be taken into careful consideration before adopting them for this purpose. The use of one animal model is another limitation and we recommend always testing efficacy of new targets in several animal models representing different risk factors for the disorder eg pharmacological, neurodevelopmental and genetic.

\section{Experimental Procedures}

\subsection{Animals}

Two separate batches of 20 and 14 adult female Lister Hooded rats obtained from Harlan UK, were used as subjects for experiments 1 and 2 respectively, 34 rats were used in total. Rats were housed in groups (4-5 rats/cage) and kept under standard laboratory conditions (room temperature $21 \pm 2^{\circ} \mathrm{C}$ and humidity $40 \%$ to $50 \%$ ) on a 12-h light/dark cycle with lights on at 0700 hours. All experiments were carried out between 9 am to $5 \mathrm{pm}$. Water was available ad libitum, but food was restricted to $10 \mathrm{~g}$ per rat per day for the duration of both experiments. Female rats were used as they have consistently demonstrated reliable performance in our laboratory in a variety of cognitive tests at all stages of the oestrus cycle (McLean et al., 2009; Sutcliffe et al., 2007), and robust deficits following our sc PCP regimen (Neill et al., 2010; 2014). There has been an unfortunate over-reliance on male rodents in animal studies in the past, a situation fortunately being rectified due to a new and overdue NIH directive (Clayton and Collins, 2014). All experiments were conducted in accordance with the UK Animals (Scientific Procedures) Act 1986 and were approved by the University of Manchester ethics committee. 


\subsection{Drugs and treatment regime}

There were two distinct experimental designs in the present study using two tasks for investigating the effect of sc PCP treatment on optimistic cognitive bias (Experiment 1) and affective bias (Experiment 2).

For experiment 1, 20 rats weighing 160-190 $\mathrm{g}$ at the start of training were randomly divided into two groups; vehicle and PCP treatment groups. Vehicle (saline; $1 \mathrm{ml} / \mathrm{kg}, \mathrm{n}=10$ ) or PCP ( $2 \mathrm{mg} / \mathrm{kg}, \mathrm{n}=10$ ) was intraperitoneally (i.p.) administered twice daily for 7 days followed by a wash-out period of 7 days. This treatment schedule occurred after the rats were trained and tested for their inherent optimistic cognitive bias. By the end of the wash-out period, rats were re-tested in order to investigate the effect of our Sc PCP treatment regimen on optimistic bias. PCP hydrochloride obtained from Sigma-Aldrich, UK, dissolved in distilled water and administered in a volume of $1 \mathrm{ml} / \mathrm{kg}$ i.p. Using the same treatment protocol as in experiment 1,14 rats weighing 200-250 $\mathrm{g}$ at the start of the experiment from a separate batch were randomly allocated to receive PCP $(n=7)$ in experiment 2, prior to training and testing. Remaining rats $(n=7)$ did not receive any treatment, hence were injection, but not handling naïve, in order to compare no injection with saline injection across experiments.

\subsection{Behavioural tests}

\subsubsection{Experiment 1: the optimistic cognitive bias test}

The chamber consisted of two identical Plexiglass boxes as described in Brydges et al. (2011) (61 x $43.5 \mathrm{~cm}$ and $45.5 \mathrm{~cm}$ high, Fig. 1) one start box and one goal box connected by a freeaccess tunnel $(10 \mathrm{~cm} \times 80 \mathrm{~cm} \times 12 \mathrm{~cm})$. Specific grade sandpapers were used to represent neutral cues which were presented in the tunnel according to the protocol described below. The goal box contained two foraging bowls, one black and one white, filled with wood shavings. Each bowl was paired with particular odour, O (eg O1: pomegranate \& raspberry, O2: aloe \& soft linen) and with a particular reward (white chocolate drop or $1 / 2$ honey nut cheerio ${ }^{\circledR}$ ) which remained constant throughout the study for each subject. The odours were purchased from the Bodyshop UK and are the same as those used in our set shifting studies where we reliably show a scPCP-induced deficit in the EDS phase (McLean et al. 2008). We have previously determined no preference or bias for these odours used. The chamber was set 
up in the experimental room which was separate to the housing area. All experiments were conducted under regular room lighting (Fig. 1).

The task of optimistic cognitive bias was conducted according to the protocol developed by Brydges and colleagues (2011) with minor modifications. In this task, there was one 'positive' and one 'less positive' event determined by the values of the outcomes. In order to create such events, two rewards with different values; white chocolate drop as high-value (HV) reward and $1 / 2$ honey nut cheerio ${ }^{\circledR}$ as low-value (LV) reward were used for positive and less positive events, respectively. In these events, each reward was associated with one neutral predictive cue, a particular grade of sandpaper (coarse or fine; P40 or P1200 Halfords, Manchester, UK) lining the floor of the tunnel. To give an example of the sequence of events, one rat always received HV reward if the tunnel was lined with coarse sandpaper and LV reward if the tunnel was lined with fine sandpaper. In both events, rats had to make an active choice in order to receive the reward (ie choose one of the 2 bowls). The rat's choice was defined as when the first digging behavior occurred. Once trained, rats then underwent optimistic cognitive bias testing. This time rats were confronted with an ambiguous trial in which they were introduced to an ambiguous stimulus, novel grade sandpaper ie a grade between the previous two (medium sandpaper; P180; Halfords, Manchester, UK) and there was no reward in the foraging bowls. The optimistic choice was defined if the rat made a choice for the bowl that was previously paired with HV reward (Fig. 2).

\section{Habituation (Phase A and B)}

Each rat was firstly handled $5 \mathrm{~min} /$ day for 5 days and both of the rewards were introduced into the rats' home cages. After introducing the rewards, rats were habituated to the chamber in cage groups for $20 \mathrm{~min} /$ day for two consecutive days (Phase A). In these sessions, each rat was placed in the start box and the tunnel was lined with a particular grade of sandpaper (P120; Halfords, Manchester, UK) which remained constant for the habituation phase, and was different from those used in subsequent training sessions. After group habituation, rats were put on restricted diet (10g food/rat/day) and the next day each rat was individually placed in the apparatus and introduced to bowl-odour-reward pairings which remained constant for each rat throughout the study. During this phase (Phase B), two foraging bowls as described above were placed either on the left or the right of the goal box. For each rat, 
each reward was specifically paired with a particular bowl-odour-location which remained consistent for that subject throughout the experiment but was counter-balanced between individual animals. Phase B lasted for 4 days ( 2 sessions/rat/day).

\section{Training (Phase $C, D$ and $E$ )}

Training consisted of three consecutive phases; C, D and E, according to the protocol of Brydges et al (2011), each of which took 3-4 days depending on the learning capabilities of each subject. Phase $\mathrm{C}$ served as a starting point for creating 'positive' or 'less positive' events as described above by representing specific neutral cues, different grades of sandpaper (Fig. 2). Each rat received 4 trials per day; 2 trials for chocolate and 2 trials for $1 / 2$ a cheerio ${ }^{\circledR}$ in a randomized trial order. In every trial, rats were individually placed in the start box and time to exit the tunnel, choose the first bowl and correct bowl containing the reward (if not chosen first) was recorded by the experimenter. In Phase C, the food rewards were placed on the surface of the digging media, in Phase $\mathrm{D}$ the rewards were buried in the digging media. The sandpaper and reward pairings were counterbalanced within each group; e.g. half of the rats had coarse sandpaper in the chocolate trial and fine sandpaper in the cheerio trial, and vice versa for the other half. In order to minimize olfactory trails, the sandpaper was changed between the groups and the apparatus was cleaned with $70 \%$ ethanol.

In phase $\mathrm{E}$, the rewards were always at the bottom of the bowls and, 1 out of 4 trials/rat/day was unrewarded (4 unrewarded trials/4 days in total) in a randomized order. Correct or incorrect responses in these unrewarded trials provided information regarding how well the subjects were trained in terms of being able to associate the cue (a particular grade of sandpaper) with the outcome (reward) in the absence of any odours provided by the rewards themselves. Subjects that were successful in making correct choices in these trials were moved on to phase F; the test of optimistic cognitive bias.

Pre-treatment testing (Phase F) of optimistic cognitive bias and sub-chronic PCP treatment effects (Phase G)

In Phase F; the unrewarded 'probe' trials (1 out of 4 trials per day) were this time paired with medium grade sandpaper as an ambiguous stimulus. The rat's choice was defined as optimistic if it chose the bowl that was previously paired with the HV reward for that subject. 
The duration of phase $\mathrm{F}$ was 3 days meaning that there were 3 probe trials in total ( 1 probe trial/day).

After completing pre-treatment testing, rats underwent PCP dosing, phase $\mathrm{G}$, as described above. Following 7 days of drug treatment, extra training sessions (phase D2) were applied during the wash-out period to ensure that rats were retaining the task. Finally, rats treated with sc PCP or vehicle were re-tested in the paradigm (phase F2) with one probe trial per day for 5 days ( 5 probe trials in total) following the 7 day washout period. It is important to emphasize that in both paradigms, rats were tested in the drug-free state, ie at least 7 days after the last PCP dose.

\subsubsection{Experiment 2: The affective bias test}

Animals were tested in a Perspex arena (length $=56 \mathrm{~cm}$, width $=36 \mathrm{~cm}$ and height $=19 \mathrm{~cm}$ ) of three compartments that we routinely use for our attentional set shifting studies (McLean et al., 2008). The arena floor was covered with sawdust. Compartments were created by two removable panels and a central divider at one third of the length of the cage. A digging bowl was placed in each of the two small compartments throughout the experiment. Rats were given access to the bowls by removing the dividers (Fig. 3).

\section{Habituation}

Rats were habituated to the experimental arena prior to the start of the experiment. As for experiment 1, a bowl was introduced to each home cage 4 days prior to training. On the fourth day, rats were allowed to freely explore the testing arena with their cage-mates in the absence of the bowls for 10 minutes for habituation. Then the bowls were placed into the arena and rats were given 5 minutes of individual habituation to the testing arena. Three half cheerios $^{\circledR}$ were then placed into each bowl to encourage the rat to attend to the bowl. This was repeated three times. Retrieval of all cheerios marked the end of the habituation session for each rat. 


\section{Training to dig}

This training session was designed to shape bowl digging behavior. Each rat was individually placed in the testing arena and presented with 2 bowls, initially containing one half cheerio ${ }^{\circledR}$. Cheerio ${ }^{\circledR}$ halves were then placed on sawdust and then gradually buried under thin and thick layers of sawdust, in a similar manner to Study 1. Each condition was repeated three times (total of 12 trials). Each training session lasted until all the cheerios ${ }^{\circledR}$ were retrieved and consumed in twelve consecutive trials.

\section{Affective bias test (general protocol)}

The affective bias test was conducted according to the protocol developed by Stuart et al., (2013). Rats treated with sc PCP or vehicle underwent the ABT. We followed a standard protocol of four pairing sessions followed by a choice test on the fifth day. In each pairing session, rats were presented with two bowls (A; days 1 and 3 or $\mathrm{B}$; days 2 and 4 and $\mathrm{C}$ all sessions), each of which was associated with an odour (Bowl A= Caliber-bean odour, Bowl $\mathrm{B}=$ Vanilla). In this experiment, Bowl A contained higher reward value $\left(\mathrm{HR}=2\right.$ cheerio ${ }^{\circledR}$ halves) and bowl B contained the lower reward value ( $\mathrm{LR}=$ one cheerio ${ }^{\circledR}$ half) throughout the pairing sessions. The second bowl (bowl C) was always unrewarded and was associated with the pomegranate odour. Each pairing session consisted of individual trials, whereby choosing the reward-containing bowl (Bowl A on days 1 and 3; Bowl B on days 2 and 4) was considered as the correct response. Upon digging in a bowl, access to the other was immediately blocked and the choices as well as digging latency were recorded in each trial. The pairing session was completed when rats correctly chose the reward containing bowl over 6 consecutive trials (Figure 4 ).

\section{The choice preference test}

On day five, rats were presented with the previously rewarded bowls (A and B) over 30 trials. At this stage of the experiment, both bowls carried equal reward value. To maintain motivation for digging, there was a one in three chance that neither bowl would be baited with reward. For each single trial, the choice of the rat and the digging latency was recorded. 


\subsection{Statistical analysis}

All behavioural scoring was conducted by an experimenter blinded to the rat's treatment group. For experiment 1; unpaired Student's t-test was used in comparisons between 2 groups (chocolate versus cheerio ${ }^{\circledR}$, sc PCP versus vehicle). Repeated measures one way ANOVA was used for within group time data comparisons in each phase. Post hoc comparisons with phase C were conducted using Dunnett's t-test. In experiment 2; student's t-test was used to analyze the percentage choice test results. To compare digging latency and number of trials to criterion for days associated with HV reward (1 and 3) and LV reward (2 and 4) between naive and sub-chronic PCP groups, repeated measures ANOVA was used. This was followed by student's t-test when appropriate. All data are represented as mean \pm S.E.M, $p<0.05$ was considered statistically significant.

\section{RESULTS}

\subsection{Optimistic bias test}

\section{Performance between the test phases}

In the present study there was a phase effect for time taken to leave the tunnel, choose the $1 \mathrm{st}$ and correct bowls. As expected, rats took longer in these parameters in the initial learning phase (phase C) compared to the other phases (Fig. 5). There was no significant difference between the two groups.

In both groups rats took significantly longer time to leave the tunnel in phase $\mathrm{C}$ compared to all other phases (phase $\mathrm{E}_{\text {vehicle; }} \mathrm{P}<0.05$, phase $\mathrm{F}_{\text {vehicle, }} \mathrm{D} 2_{\text {vehicle }}, \mathrm{F} 2_{\text {vehicle; }} \mathrm{P}<0.01$, phase $\mathrm{D}$ PCP, phase $E_{\text {PCP; }}$ D2 PCP, F2 PCP; $\mathrm{P}<0.01$, phase F PCP; $\mathrm{P}<0.05$ ) (Fig. 5A).

Time taken to choose the 1st bowl started to decrease after phase D and remained significantly faster in the other consecutive phases compared to phase $\mathrm{C}$ in both groups (phase $\mathrm{E}_{\mathrm{PCP}}$; $<0.05$, phase $\mathrm{F}_{\text {vehicle \& PCP; }} \mathrm{p}<0.05$, phase $\mathrm{D} 2_{\text {vehicle }} \mathrm{p}<0.01$, phase D2 ${ }_{\mathrm{PCP} ;} \mathrm{p}<0.001$, phase F 2 vehicle \& PCP; $\mathrm{p}<0.001)$ (Fig.5B).

In both groups, time to choose the correct bowl gradually decreased compared to the initial phase $\mathrm{C}$, this decrease was statistically significant throughout all phases except phase D for 
the PCP group (phase $\mathrm{D}$ vehicle; $\mathrm{p}<0.01$, phase $\mathrm{E}-\mathrm{F} 2_{\text {vehicle; }} \mathrm{p}<0.001$, phase $\mathrm{E} \& \mathrm{~F}$ PCP; $\mathrm{p}<0.01$, phase D2 \& F2 2 PC; $<<0.001)$ (Fig. 5C).

\section{Experiment 1: Comparisons between overall chocolate versus cheerio trials}

Rats took significantly less time to choose the first $(p<0.01)$ and correct bowl $(p<0.01)$, in chocolate trials compared with cheerio ${ }^{\circledR}$ trials, demonstrating a preference for the HV reward. Time to exit the tunnel was not statistically different between the two rewards $(\mathrm{p}=0.27)$ (Fig. $6)$.

\section{Experiment 1: The effect of sc PCP on optimistic choices}

Before the treatment, there was no difference in the number of optimistic choices between the two groups $(\mathrm{p}=0.79)$ Cognitive bias was in favour of optimism since each group made optimistic choices in 2 out of 3 probe trials on average (Fig. 7A). After the treatment, the mean number of optimistic choices was shown to be significantly decreased in the sc PCP group compared to the vehicle group $(\mathrm{p}<0.01)$ (Fig. 7B). The vehicle group remained in the optimistic state (optimistic choices, 2.7/5) after the treatment however, the PCP group had markedly reduced optimistic choices, 1.3/5.

\subsection{Affective Bias test}

\section{Effect of sc PCP in the affective bias choice test}

Results of the paired t-test analysis on choice test data in the ABT revealed that naïve rats formed a significant positive affective bias towards bowl A, which contained the higher reward, ie a whole cheerio $(\mathrm{t}=2.71, \mathrm{p}<0.05)$. In marked contrast, rats treated with PCP did not form a preference for either bowl, suggesting the absence of a positive affective state $(\mathrm{t}=0.84, \mathrm{p}=0.41),($ Fig. 8). 


\section{Effect of sc PCP on digging latency and trials to criterion; comparison between HV reward}

\section{(days 1and 3) and LV reward (days 2 and 4) sessions}

Investigation of effects of sc PCP on the number of trials to criterion and digging latency (Fig. 9) associated with HV and LV reward trials revealed no significant effects of HV versus LV reward or sc PCP versus no treatment.

\section{Discussion}

In fulfillment of our aims in this study, we evaluated the validity of two novel behavioral tests for exploring affective state and anticipation deficits of relevance to schizophrenia. We have demonstrated that sc PCP treatment followed by washout, induces a deficit in anticipatory behaviour (experiment 1) and affective processing (experiment 2) shown as negative evaluation of an ambiguous situation and lack of preference for a high value reward. Briefly, in experiment 1, rats gave optimistic responses (foraging the bowl that was previously paired with the HV reward) in the presence of an ambiguous stimulus before sc PCP treatment. After treatment however, sc PCP-treated rats exhibited pessimistic responses, in other words, they showed anticipatory deficits compared to sc vehicle-treated rats. Another important finding here was that chocolate was of a higher value than cheerios in our design, confirming the original results of Brydges et al. (2011). Rats were significantly faster to choose the first and correct bowl in chocolate compared to cheerio trials demonstrating enhanced motivation for the higher value reward, as anticipated.

In the present study, we adapted the optimistic cognitive bias protocol developed by Brydges et al. (2011) because it provides a number of methodological advantages compared with previous studies in this area. First of all, unlike previous studies using go/no-go tasks (Bateson and Matheson, 2007; Harding et al., 2004), the Brydges task is an active choice paradigm in which rats have to make an active response to both events, considered to provide clearer insight into the rats' behaviour in this paradigm (ie. was it an active choice and therefore, decision?) Another important feature of Brydges's design is that they do not use punishment to create a 'negative' event unlike previous studies (Bateson and Matheson, 2007; Burman et al., 2008; Harding et al., 2004) rather they created a 'positive' and 'less positive' event dependent upon the value of the reward. This approach avoids the considerable disadvantages of using negative events in animal studies. Exposure to repeated 
punishments would be expected to influence affective state of the animals apart from the model itself (in our case sc PCP model) in addition to possibly interfering with the reaction/response of the animals to the task. This point becomes even more important when using a design requiring an extended training time as required in the optimistic bias task used here. Alternatively, such a design better reflects real life situations which normally require motivation or positive anticipation of a specific outcome in daily events. This positive anticipatory system is known to be disrupted in people with a negative affective state such as those with persistent negative symptoms. It is unlikely that our finding of reduced anticipatory motivation induced by sc PCP is attributable to known cognitive deficits induced by this treatment regimen (see Neill et al. 2010 and 2014 for reviews). Following treatment, each rat remained in training until it made a correct response in the unrewarded probe trial, showing that the rats were still able to make a correct association between a particular grade of sandpaper and the reward. Only after being successful, were rats re-tested for their optimistic cognitive bias. Therefore, any learning deficits induced by sc PCP in this procedure would have been apparent at this stage. It is possible of course that we may not have detected subtle cognitive deficits, due to our experimental design in which rats were fully trained prior to the sc PCP treatment regimen. However in our operant reversal learning task, rats are fully trained prior to sc PCP treatment and still show a specific and selective reversal learning deficit (McLean et al. 2009; 2011). It is important to note that in this case we started to re-train rats in the washout period due to time constraints, unlike in our standard protocol where we leave at least 7 days post-PCP before training or testing the animals. One disadvantage of this paradigm in its current form is that it requires a relatively lengthy procedure (approx. 40 days in total) and we predict that it will be difficult to re-use the animals to test several pharmacological agents, rather like in the attentional set shifting task. Once the rats have been exposed to medium grade sandpaper, it stops being an ambiguous stimulus and either new stimuli will be required or new animals, not in compliance with the 3Rs (Replacement, Reduction and Refinement, www.nc3rs.org.uk/the-3rs). In its current state therefore, this will not provide a high throughput test. However, with further development, we believe that these obstacles will almost certainly be overcome.

In the second experiment, non-injected rats showed sensitivity to changes in the absolute reward value (choosing the bowl with higher reward value), while this sensitivity was absent in the sc PCP treated rats. Accordingly we concluded that there is reduced affect (affective 
blunting), in the sc PCP-treated rats. The affective bias test is heavily dependent on learning the association between an odour and the reward-containing bowl. Given that our data shows no difference between the groups in number of trials to criterion, it could be concluded that there is no difference between the control and sc PCP group in learning the reward-odour association. This is further confirmed by other paradigms in regular use in our lab, such as attentional set-shifting, in which rats sub-chronically treated with PCP retain the ability to form a reward-odour or digging medium association (McLean et al., 2008, 2011).

Recently, Lydall and colleagues showed that sub-chronic treatment with PCP does not reduce the value of a reward which is of high value for rats (sucrose) (Lydall et al., 2010). They conclude (as do we, reviewed in Neill et al. 2014) that scPCP does not induce anhedonia per se. As discussed in the Introduction, anhedonia is not considered a key component of negative symptoms of schizophrenia. Therefore, the indifference of the sub-chronic PCP treated rats towards reward value cannot be accounted for by a reduction in valuation of the $\mathrm{HV}$ reward. Also the significant positive bias formed by the control rats towards the reward with higher value shows that this reward carries high enough value to be distinct from the other reward. The manifested deficit in sc PCP rats, therefore, might be better explained by the influence of sc PCP treatment on brain regions involved in affective processing.

The role of the anterior cingulate cortex (ACC) in regulating emotions in animals and humans (Drevets and Savitz, 2008) is well established. Based on imaging and postmortem studies, the ACC is abnormal in terms of activity (Adams and David, 2007), regional morphology (reduced gray matter density) (Bouras et al., 2001) and neurobiology (reduced Nacetylaspartate) (Ende et al., 2000) in patients with schizophrenia. Sub-chronic treatment with PCP induces similar abnormalities including a significant reduction of the number of parvalbumin containing interneurons in hippocampus (Abdul-Monim et al., 2007) and a reduction of the grey matter density in prefrontal cortex as well as amygdala, hippocampus and ACC (Barnes et al. 2014b). The ACC also forms extensive connections with the amygdala (important for emotional processing) (Aleman and Kahn, 2005) and with the prefrontal cortex (important for cognition) (Stevens et al., 2011). These interconnections allow for appropriate appraisal of stimuli and regulation of the appropriate affective response. Given that these interconnections are reciprocal between these structures, it can be concluded that affect and cognition can influence one another. This reciprocal interaction between cognition and affect is the principle to which both the optimistic bias task and the affective 
bias test both adhere (manipulating cognition to influence affect in the optimistic bias task and manipulating affect to influence cognition in the affective bias task). Based on current evidence, we can speculate that the deficits seen in the affective bias and optimistic cognitive bias tests demonstrated here are due to the fact that sc PCP treatment strongly influences this reciprocal relationship by affecting functional connectivity. The way in which the functional connectivity between these regions is affected by sc PCP treatment however remains to be ascertained.

Our study has certain limitations. Across our two experiments the rewards of choice, especially for $\mathrm{HV}$ rewards were inconsistent with one another. Although this can be explained by our intention to keep the protocols as similar to the original studies as possible, the inconsistency may have compromised our conclusions. Indeed, we observed that rats consumed chocolate drops faster than cheerios and were more motivated to seek chocolate over cheerios in the optimistic cognitive bias task. However, this was not necessarily the case with the affective bias task which did not require rats to expend much effort to retrieve the food reward. In future, it will be important to conduct the ABT using chocolate as the high value reward. In both tasks the size of the deficit produced by sc PCP is rather small which will make assessment of its reversal rather difficult. Currently and following discussion with the Robinson laboratory, we are in the process of refining the ABT protocol to include 3 pairing sessions. This would allow us to strengthen the association of stimulus with reward. Moreover, the tests described above rely on presumed alterations in affective state to induce cognitive bias using environmental manipulations. However, the true influence of such manipulations on the valence of affective state is rather ambiguous and the pharmacological validation of these tests remains limited (Anderson et al., 2013; Mendl et al., 2009; Stuart et al., 2013) In comparison to the optimistic bias task, the ABT has been more thoroughly validated by pharmacological studies, supporting the validity of this test for measuring drug effects on affective state (Stuart et al., 2013). Pharmacological manipulation in the optimistic bias test remains to be studied. Indeed pharmacological validation of both of these paradigms as tests for negative affect of relevance to negative symptoms in schizophrenia in our sc PCP model will be our next step.

An intact emotional/affective regulatory system and appropriate processing of such information plays an important role in social interactions and is essential for effective communication. Deficits in this ability, as seen in schizophrenia patients, particularly those 
with persistent negative symptoms therefore, greatly compromise their social behaviour and functional outcomes. Treatments for these and other negative symptoms remain a major problem as this cluster of symptoms are unresponsive to current medication (Blanchard and Cohen, 2006; Kirkpatrick et al., 2006). Therefore, accurate investigation of emotional/affective state in animals is of great clinical importance, as it provides a tool for identifying novel drug targets and therapeutic compounds for treating anticipatory motivation and affective bias, aspects of negative symptoms. Our findings show for the first time that anticipatory deficits and negative affect, core negative symptoms of schizophrenia, are present in our sc PCP model and can be investigated using these two behavioral paradigms. It is our opinion that further development of these novel paradigms will provide a tool to investigate negative affect of relevance to schizophrenia in animals. This will enhance our understanding of the neurobiology of such deficits with the ultimate aim of developing new therapeutic strategies for these particular aspects of negative symptoms.

\section{Role of funding source}

This work was funded by a BAP (British Association for Psychopharmacology) in vivo vacation studentship awarded to Nazanin Doostdar and by the University of Manchester.

\section{Contributors}

Jo Neill participated in the study design, conceived, managed and supervised the experiments and assisted with interpretation of the results. Ceren Sahin and Nazanin Doostdar conducted the experiments and data analysis, participated in the study design and assisted with interpretation of the results. All authors participated equally in preparing the manuscript for publication.

\section{Conflict of Interest}

Jo Neill has received expenses to attend conferences and fees for lecturing and consultancy work (including attending advisory boards) from the manufacturers of various antipsychotic drugs. Jo Neill is an employee of the University of Manchester (UoM). Nazanin Doostdar is a 
postgraduate student at UoM. Ceren Sahin is a postgraduate student at the University of Marmara in Turkey.

\section{References}

Abdul-Monim, Z., Neill, J.C., Reynolds, G.P., 2007. Sub-chronic psychotomimetic phencyclidine induces deficits in reversal learning and alterations in parvalbuminimmunoreactive expression in the rat. J. Psychopharmacol. 21, 198-205.

Adams, R., David, A.S., 2007. Patterns of anterior cingulate activation in schizophrenia: a selective review. Neuropsychiatr. Dis. Treat. 3, 87-101.

Aleman, A., Kahn, R.S., 2005. Strange feelings: Do amygdala abnormalities dysregulate the emotional brain in schizophrenia? Prog. Neurobiol. 77, 283-298.

Anderson, M.H., Munafo, M.R., Robinson, E.S.J., 2013. Investigating the psychopharmacology of cognitive affective bias in rats using an affective tone discrimination task. Psychopharmacology 226, 601-613.

Arango, C., Garibaldi, G., Marder, S.R., 2013. Pharmacological approaches to treating negative symptoms: A review of clinical trials. Schizophr. Res. 150, 346-352.

Barnes, S.A., Der-Avakian, A., Markou, A., 2014a. Anhedonia, avolition, and anticipatory deficits: assessments in animals with relevance to the negative symptoms of schizophrenia. Eur. Neuropsychopharm. 24, 744-758.

Barnes, S.A., Sawiak, S.J., Caprioli, D., Jupp, B., Buonincontri, G., Mar, A.C., Harte, M.K., Fletcher, P.C., Robbins, T.W., Neill, J.C., Dalley, J.W., 2014b. Impaired limbic corticostriatal structure and sustained visual attention in a rodent model of schizophrenia. Int. J. Neuropsychopharm. 18, 1-12. 
Bateson, M., Matheson, S.M., 2007. Performance on a categorisation task suggests that removal of environmental enrichment induces 'pessimism' in captive European starlings (Sturnus vulgaris). Anim. Welfare. 16, 33-36.

Blanchard, J.J., Cohen, A.S., 2006. The structure of negative symptoms within schizophrenia: Implications for assessment. Schizophr Bull. 32, 238-245.

Bouras, C., Kovari, E., Hof, P.R., Riederer, B.M., Giannakopoulos, P., 2001. Anterior cingulate cortex pathology in schizophrenia and bipolar disorder. Acta. Neuropathol. 102, 373-379.

Brilot, B.O., Asher, L., Bateson, M., 2010. Stereotyping starlings are more 'pessimistic'. Anim. Cog. 13, 721-731.

Brilot, B.O., Normandale, C.L., Parkin, A., Bateson, M., 2009. Can we use starlings' aversion to eyespots as the basis for a novel 'cognitive bias' task? Appl. Anim. Behav. Sci. 118, 182190.

Brydges, N.M., Leach, M., Nicol, K., Wright, R., Bateson, M., 2011. Environmental enrichment induces optimistic cognitive bias in rats. Anim. Behav. 81, 169-175.

Burman, O.H.P., Parker, R.M.A., Paul, E.S., Mendl, M., 2008. Sensitivity to reward loss as an indicator of animal emotion and welfare. Biol. Letters. 4, 330-333.

Clayton, J.A., Collins, F.S., 2014. NIH to balance sex in cell and animal studies. Nature 509, 282-283.

Davis, M.C., Horan, W.P., Marder, S.R., 2014. Psychopharmacology of the negative symptoms: Current status and prospects for progress. Eur. Neuropsychopharm. 24, 788-799.

Drevets, W.C., Savitz, J., 2008. The Subgenual Anterior Cingulate Cortex in Mood Disorders. Cns. Spectrums. 13, 663-681. 
Ende, G., Braus, D.F., Walter, S., Weber-Fahr, W., Soher, B., Maudsley, A.A., Henn, F.A., 2000. Effects of age, medication, and illness duration on the N-acetyl aspartate signal of the anterior cingulate region in schizophrenia. Schizophr. Res. 41, 389-395.

Foussias, G., Agid, O., Fervaha, G., Remington, G., 2014. Negative symptoms of schizophrenia: clinical features, relevance to real world functioning and specificity versus other CNS disorders. Eur Neuropsychopharm. 24, 693-709.

Foussias, G., Siddiqui, I., Fervaha, G., Agid, O., Remington, G., 2015. Dissecting negative symptoms in schizophrenia: Opportunities for translation into new treatments. J. Psychopharmacol. 29, 116-126.

Fusar-Poli, P., Papanastasiou, E., Stahl, D., Rocchetti, M., Carpenter, W., Shergill, S., McGuire, P., 2015. Treatments of Negative Symptoms in Schizophrenia: Meta-Analysis of 168 Randomized Placebo-Controlled Trials. Schizophr. Bull. 41, 892-899.

Grayson, B., Leger, M., Adamson, L., Harte, M., Neill, JC., 2015. Assessment of diseaserelated cognitive impairments using the novel object recognition (NOR) task in rodents. Behav. Brain.Res. 285, 176-193.

Gururajan, A., Taylor, D.A., Malone, D.T., 2010. Current pharmacological models of social withdrawal in rats: relevance to schizophrenia. Behav. Pharmacol. 21, 690-709.

Harding, E.J., Paul, E.S., Mendl, M., 2004. Animal behavior - Cognitive bias and affective state. Nature 427, 312-312.

Kirkpatrick, B., Fenton, W.S., Carpenter, W.T., Marder, S.R., 2006. The NIMH-MATRICS consensus statement on negative symptoms. Schizophr. Bull. 32, 214-219.

Knuesel, I., Chicha, L., Britschgi, M., Schobel, S.A., Bodmer, M., Hellings, J.A., Toovey, S., Prinssen, E.P., 2014. Maternal immune activation and abnormal brain development across CNS disorders. Nat. Rev. Neurol. 10, 643-660. 
Kring, A.M., Barch, D.M., 2014. The motivation and pleasure dimension of negative symptoms: Neural substrates and behavioral outputs. Eur. Neuropsychopharm. 24, 725-736.

Leger. M., Alvaro G., Large C., Harte, M., Neill, JC., 2015. AUT6, a novel Kv3 channel modilator, reverses cognitive and neurobiological dysfunction in a rat model of relevance to schizophrenia symptomatology. Eur. Neuropsychopharm 25, S480.

Lydall, E.S., Gilmour, G., Dwyer, D.M., 2010. Analysis of licking microstructure provides no evidence for a reduction in reward value following acute or sub-chronic phencyclidine administration. Psychopharmacology 209, 153-162.

Matheson, S.M., Asher, L., Bateson, M., 2008. Larger, enriched cages are associated with 'optimistic' response biases in captive European starlings (Sturnus vulgaris). Appl.Anim. Behav. Sci 109, 374-383.

McLean, S.L., Beck, J.R., Woolley, M.L., Neill, J.C., 2008. A preliminary investigation into the effects of antipsychotics on sub-chronic phencyclidine-induced deficits in attentional setshifting in female rats. Behav.Brain.Res.189, 152-158.

McLean, S.L., Grayson, B., Idris, N.F., Lesage, A.S., Pemberton, D.J., Mackie, C., Neill, J.C., 2011. Activation of alpha7 nicotinic receptors improves phencyclidine-induced deficits in cognitive tasks in rats: implications for therapy of cognitive dysfunction in schizophrenia. Eur. Neuropsychopharm. 21, 333-343.

McLean, S.L., Idris, N.F., Woolley, M.L., Neill, J.C., 2009. D-1-like receptor activation improves PCP-induced cognitive deficits in animal models: Implications for mechanisms of improved cognitive function in schizophrenia. Eur. Neuropsychopharm. 19, 440-450.

Meltzer, H.Y., 2013. Update on typical and atypical antipsychotic drugs. Annu. Rev. Med. 64, 393-406. 
Mendl, M., Burman, O.H.P., Parker, R.M.A., Paul, E.S., 2009. Cognitive bias as an indicator of animal emotion and welfare: Emerging evidence and underlying mechanisms. Appl. Anim. Behav. Sci. 118, 161-181.

Morris, B.J., Cochran, S.M., Pratt, J.A., 2005. PCP: from pharmacology to modelling schizophrenia. Curr. Opin Pharmacol. 5, 101-106.

Moser, P., 2014. Evaluating negative-symptom-like behavioural changes in developmental models of schizophrenia. Eur. Neuropsychopharm 24, 774-787.

Neill, J.C., Barnes, S., Cook, S., Grayson, B., Idris, N.F., McLean, S.L., Snigdha, S., Rajagopal, L., Harte, M.K., 2010. Animal models of cognitive dysfunction and negative symptoms of schizophrenia: focus on NMDA receptor antagonism. Pharmacol. \& Ther. 128, 419-432.

Neill, J.C., Harte, M.K., Haddad, P.M., Lydall, E.S., Dwyer, D.M., 2014. Acute and chronic effects of NMDA receptor antagonists in rodents, relevance to negative symptoms of schizophrenia: a translational link to humans. Eur. Neuropsychopharm. 24, 822-835.

Stevens, F.L., Hurley, R.A., Taber, K.H., 2011. Anterior Cingulate Cortex: Unique Role in Cognition and Emotion. J. Neuropsych. 23, 120-125.

Stuart, S.A., Butler, P., Munafo, M.R., Nutt, D.J., Robinson, E.S., 2013. A translational rodent assay of affective biases in depression and antidepressant therapy. Neuropsychopharmacology. 38, 1625-1635.

Sutcliffe, J.S., Marshall, K.M., Neill, J.C., 2007. Influence of gender on working and spatial memory in the novel object recognition task in the rat. Behav. Brain. Res 177, 117-125.

Tsapakis, E.M., Dimopoulou, T., Tarazi, F.I., 2015. Clinical management of negative symptoms of schizophrenia: An update. Pharmacol. \& Ther. 153, 135-147. 
Wilson, C.A., Koenig, J.I., 2014. Social interaction and social withdrawal in rodents as readouts for investigating the negative symptoms of schizophrenia. Eur. Neuropsychopharm.24, 759-773.

Young, J.W., Powell, S.B., Risbrough, V., Marston, H.M., Geyer, M.A., 2009. Using the MATRICS to guide development of a preclinical cognitive test battery for research in schizophrenia. Pharmacol Ther. 122, 150-202.

\section{Figure Captions}

Figure 1. A schematic diagram of the apparatus used in experiment 1.

Figure 2. A schematic diagram showing an example of the pairings used in chocolate (HV) and cheerio (LV) trials. The probe trial is the test for optimistic cognitive bias. Each bowl containing a specific reward is paired with a particular odour (not depicted here) (HV: Highvalue, LV: Low-value).

Figure 3. A schematic diagram of the testing arena used in experiment 2, clay bowls are separated by clear Perspex dividers from the larger compartment and from each other.

Figure 4. Schematic diagram showing the protocol for experiment 2: High vs. Low reward value, Bowl A contains two cheerio halves (HV), Bowl B contains one cheerio half (LV), Bowl C contains no reward. Rats are presented with Bowls $\mathrm{A}$ and $\mathrm{C}$ on days 1 and 3, and Bowls B and C on days 2 and 4. On the fifth day, rats are presented with Bowls A and B, both of which contain equal reward value.

Figure 5 A-C. Time to exit the tunnel (A), choose the $1^{\text {st }}(\mathrm{B})$ and correct bowl (C) throughout phases C-F2 of Experiment 1. Data are expressed as \pm S.E.M time taken in seconds (s). Data were analyzed by one-way ANOVA (repeated measures). Significant effects: ${ }^{* \#} \mathrm{P}<0.05,{ }^{\# * *} \mathrm{P}<0.01,{ }^{\# * * * *} \mathrm{P}<0.001$ significant difference compared with Phase $\mathrm{C}$, *=vehicle group, \#=PCP group, Dunnett's t-test ( $\mathrm{n}=10 /$ group). PCP (2 mg/kg i.p.) or vehicle (0.9\% saline i.p.) was administered twice daily for 7 days followed by 7 days washout.

Figure 6. Time to exit tunnel, to choose the $1^{\text {st }}$ and correct bowl during chocolate (HV) versus cheerio (LV) trials. Data are expressed as mean \pm S.E.M time (s) and analysed by 
unpaired student's t-test ( $\mathrm{n}=10 /$ group). ${ }^{*} * \mathrm{P}<0.01$; significant difference between chocolate and cheerio trials. (HV: High-value, LV: Low-value).

Figure 7. The mean number of optimistic choices during probe trials in pre-treatment (A) and post-treatment (B) testing phases. PCP (2 mg/kg i.p.) or vehicle ( $0.9 \%$ saline i.p.) was administered twice daily for 7 days followed by 7 days washout. Data are expressed as mean \pm S.E.M and analysed by student's t-test (unpaired). $* * \mathrm{P}<0.01$ significant difference between the vehicle and sc PCP group ( $\mathrm{n}=10$ /group). PCP $(2 \mathrm{mg} / \mathrm{kg}$ i.p.) or vehicle $(0.9 \%$ saline i.p.) was administered twice daily for 7 days followed by 7 days washout

Figure 8. Percentage choice response of vehicle and sc PCP rats on the choice test day. Data are shown as mean \pm SEM and analysed by Student's t-test $(\mathrm{n}=7 /$ group). $* \mathrm{P}<0.05$ significant increase in \% selection of high versus low value reward paired bowl. PCP (2 $\mathrm{mg} / \mathrm{kg}$ i.p.) or vehicle ( $0.9 \%$ saline i.p.) was administered twice daily for 7 days followed by 7 days washout.

Figure 9. The effect of sc PCP treatment on the number of trials to criterion (A) and digging latency (B) between HV reward days (1 and 3) and LV reward days (2 and 4). ( $\mathrm{n}=7 /$ group). PCP (2 mg/kg i.p.) or vehicle (0.9\% saline i.p.) was administered twice daily for 7 days followed by 7 days washout.

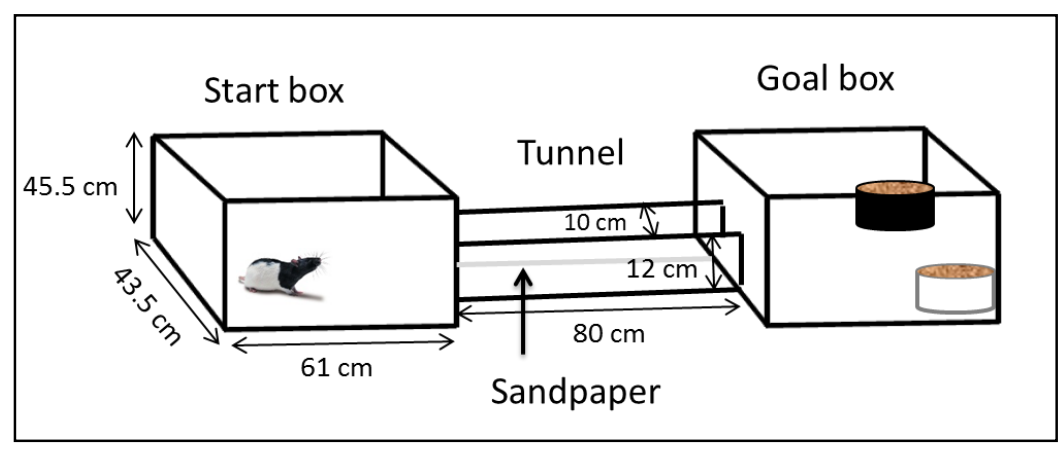

Figure 1. 

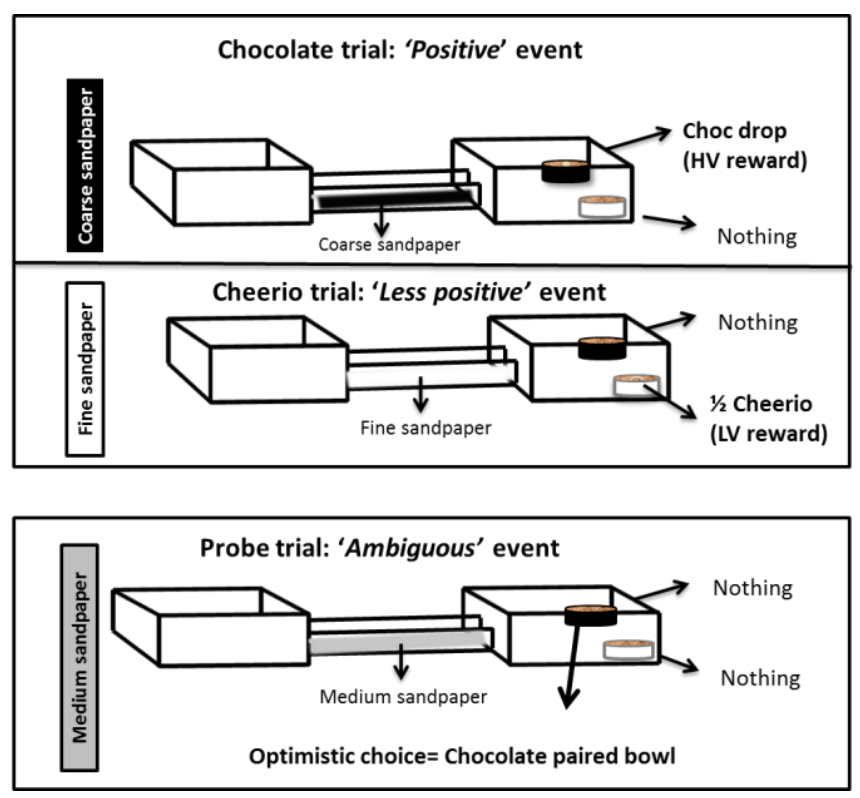

Figure 2. 


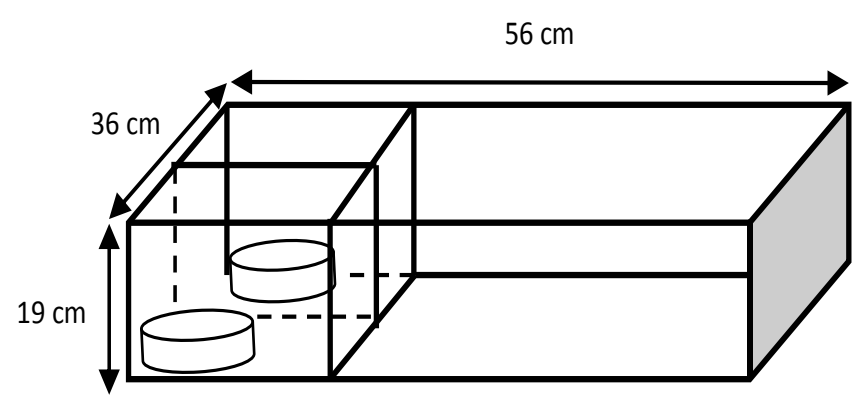

Figure 3.

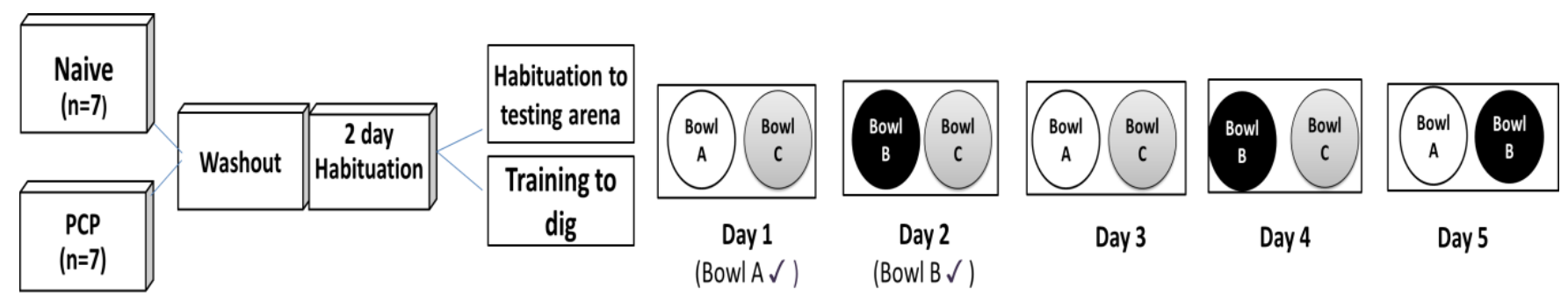

\section{Figure 4.}

Time to exit the tunnel

(A)

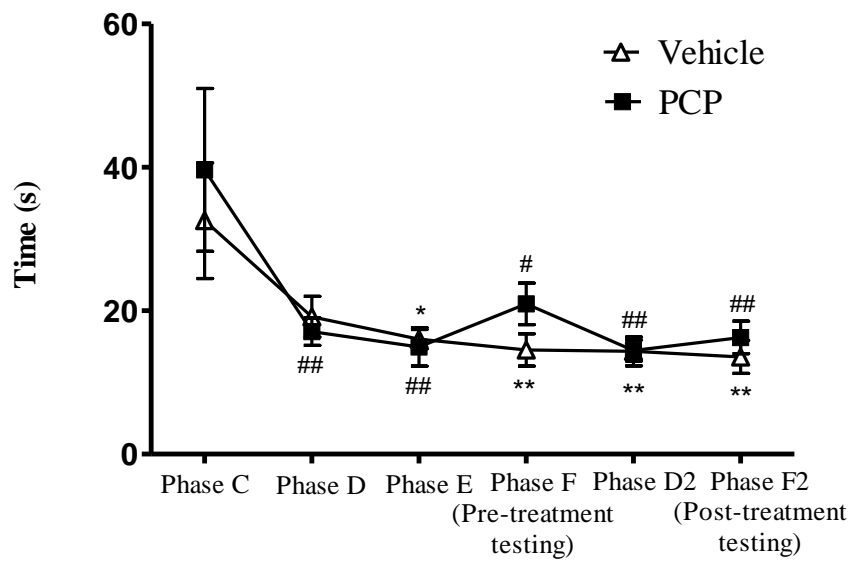


Time to choose 1st bowl

(B)

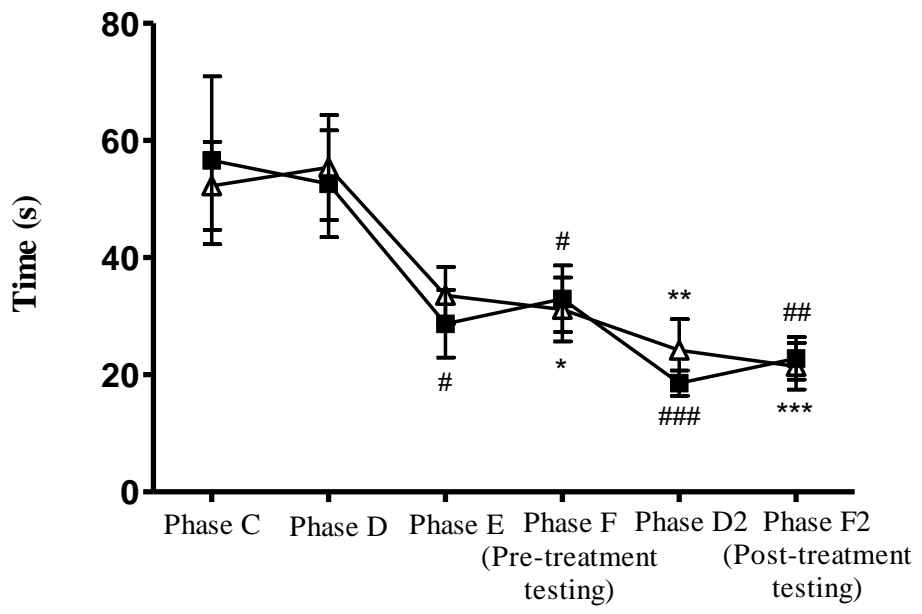

Time to choose correct bowl

(C)

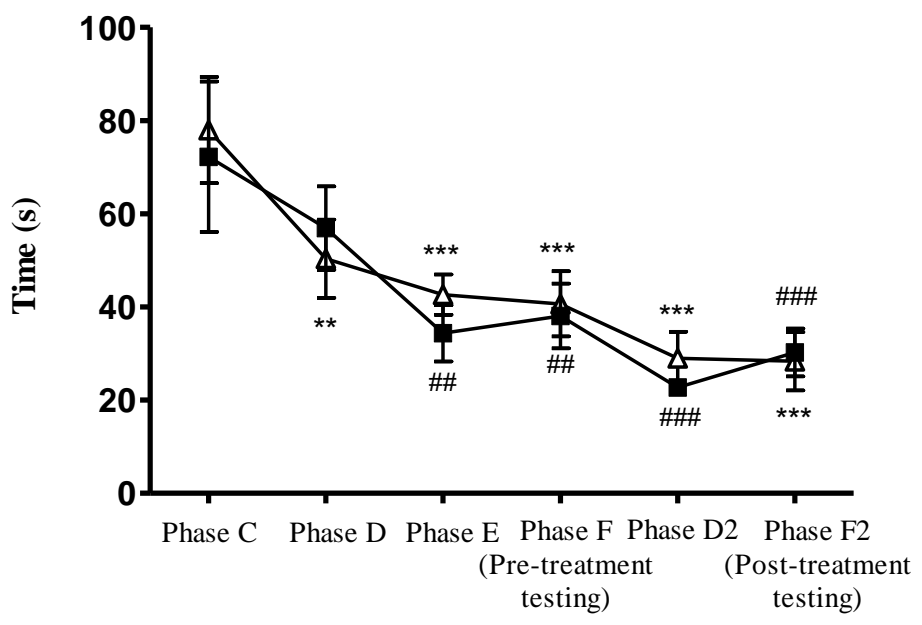

Figure 5 


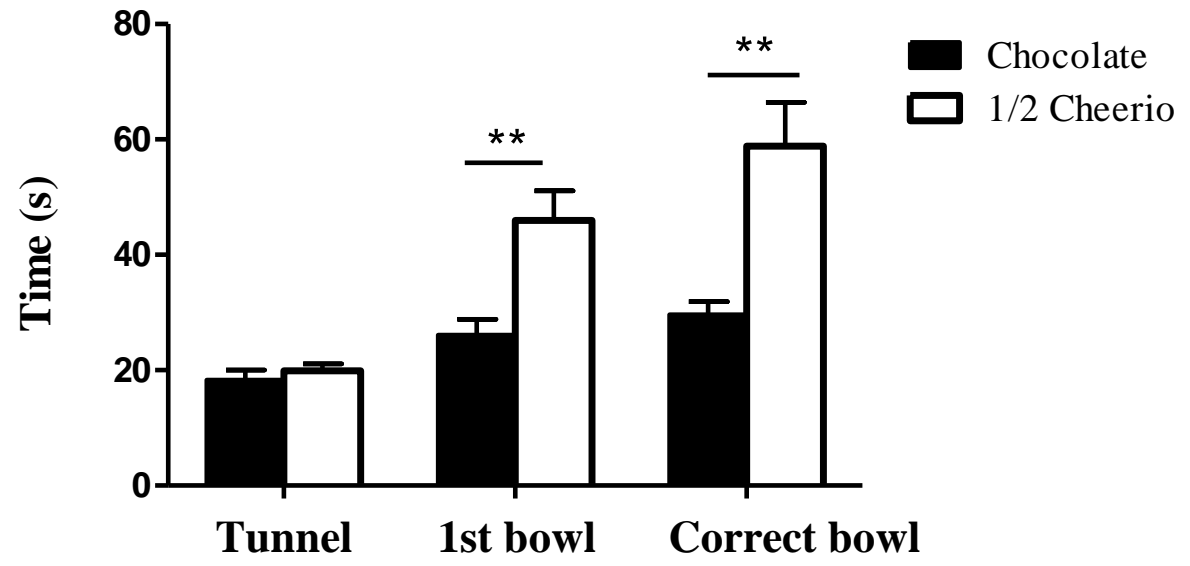

Figure 6

A

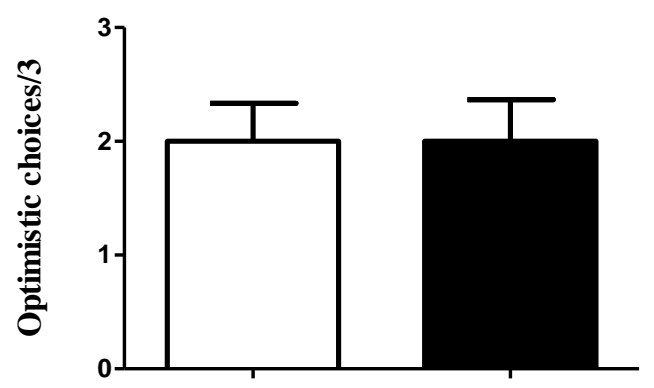

Pre-treatment (Phase F)
B

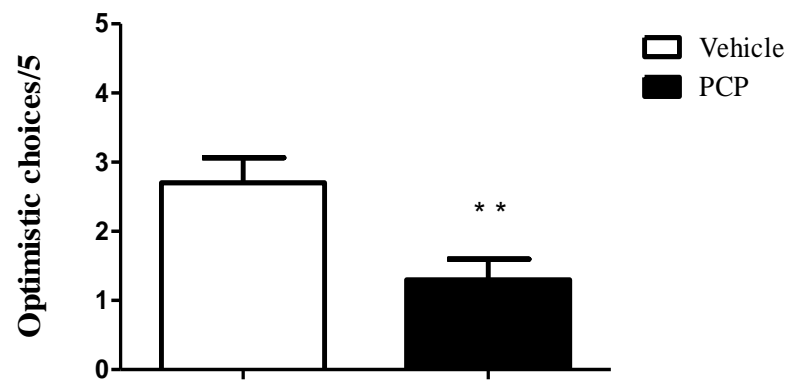

Post-treatment (Phase F2)

Figure 7. 


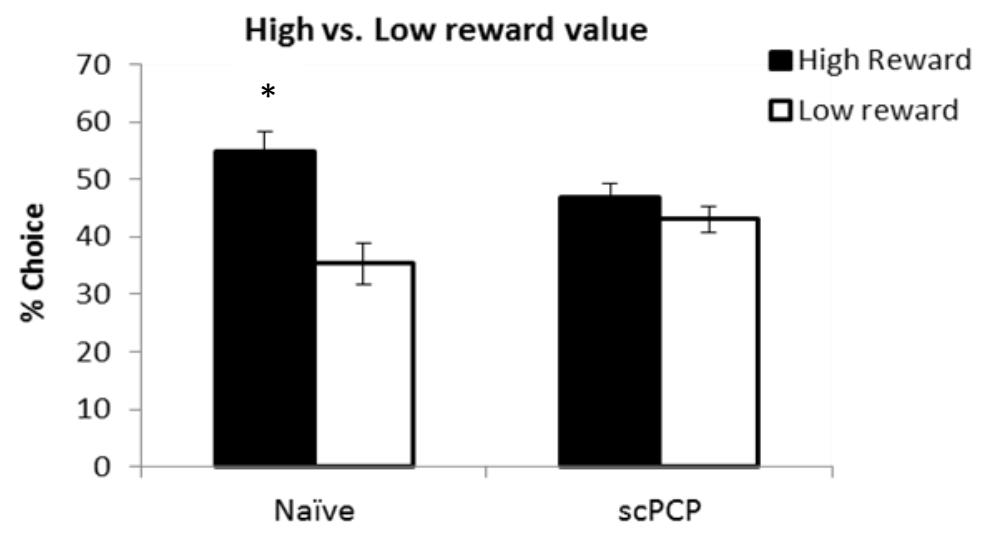

Figure 8

A

Effect of PCP on number of trials to criterion

B
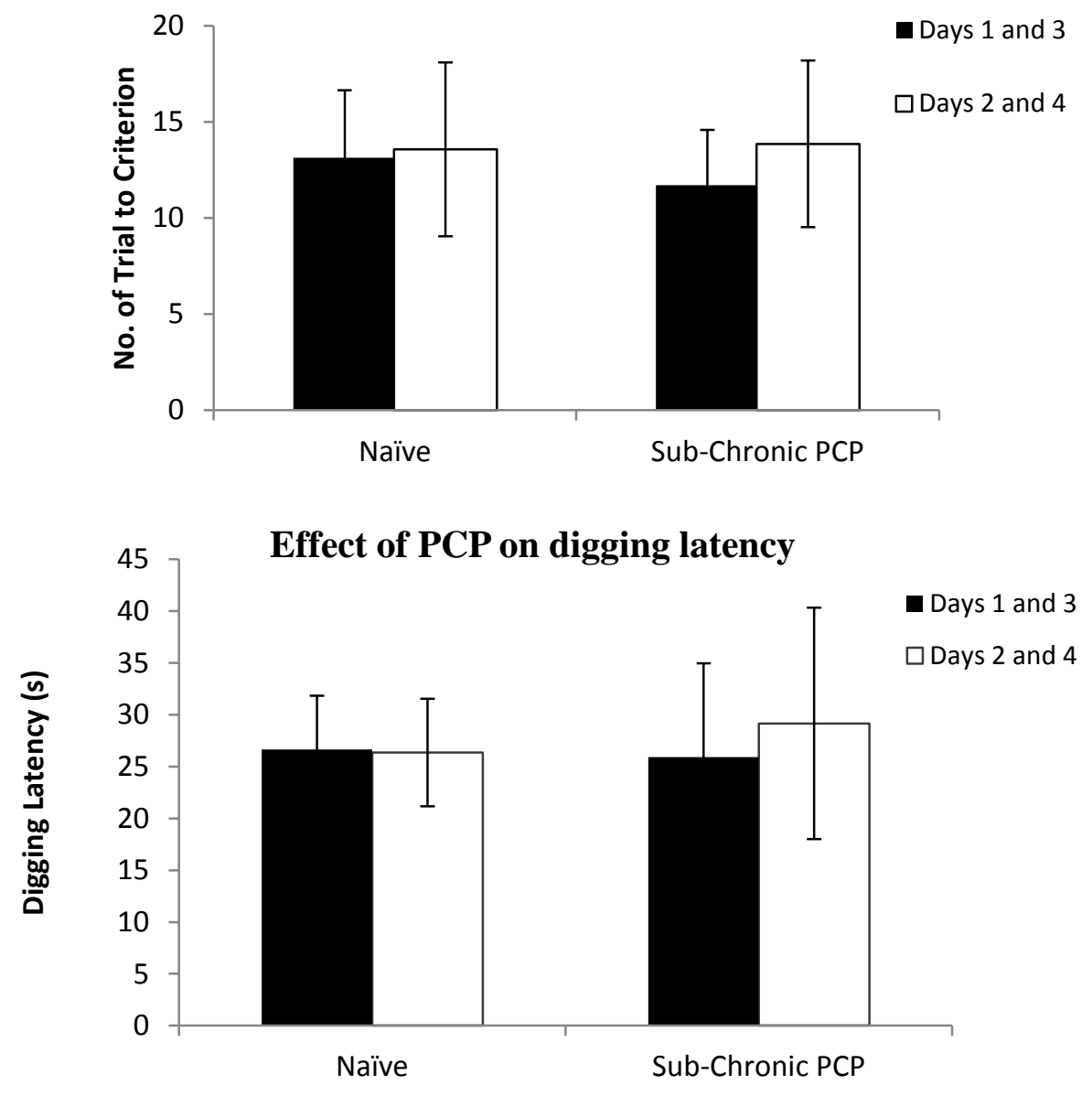

Figure 9. 